\title{
Angiomyofibroblastoma of the Vagina
}

\author{
Danijel Bursać ${ }^{1 *}$, Mario Podgajski² and Dejana Lučić ${ }^{3}$ \\ ${ }^{1}$ Department of Obstetrics and Gynecology, University Hospital Merkur, Croatia \\ ${ }^{2}$ Department of Pathology, University Hospital Merkur, Croatia \\ ${ }^{3}$ Department of Obstetrics and Gynecology, General Hospital Vinkovci, Croatia
}

Submission: January 10, 2018; Published: February 06, 2018

*Corresponding author: Danijel Bursać, Department of Obstetrics and Gynecology, University Hospital Merkur, Zajčeva 19, 10000 Zagreb, Croatia, Tel. +38598833488; Fax: +38512431 402; Email: danijel.cigra@gmail.com

\begin{abstract}
Angiomyofibroblastoma (AMF) is a rare and benign soft-tissue tumor. It belongs to the group of genital mesenchymal tumors mostly occurring in lower genital tracts of young to middle-aged women [1]. These tumors occur primarily on vulva, perineum, vagina, uterine cervix [24] and the inguinoscrotal regions of men [5]. There are only few cases described with primary occurrence in the pelvis or retroperitoneum [6,7]. It should be differentiated from aggressive angiomiyxoma, cellular angiofibroma, and other myxoid tumors of genital tract [6,8], in which radical surgical treatment is needed. Rare recurrences of AMF after excision have been reported in the literature [9]. We present a case of a polypoid, firm, pedunculated and mobile vaginal mass that originated from left lateral vaginal wall, diagnosed to be vaginal angiomyofibroblastoma.
\end{abstract}

Keywords: Angiomyofibroblastoma; Rare tumor; Soft-tissue tumor; Genital mesenchymal tumors

Abreviations: AMF: Angiomyofibroblastoma; CT: Computed Tomography; AAM: Aggressive Angiomyxoma; IHC: Immunohistochemical; DFML: Dendritic Fibromyxolipoma; IMT: Inflammatory Myofibroblastic Tumor

\section{Introduction}

A 48-year-old, G2, P2, Caucasian women presents in our outpatient department, with painless mass protruding from vagina. Otherwise, she had no gynaecological problems except uncomfortable feeling present in lower abdomen. On examination, polypoid, firm, pedunculated and mobile vaginal mass $(5 \times 5 \mathrm{~cm})$ was found originating from left lateral vaginal wall. The mass was rapidly growing during past three months. She was examined three months earlier and mass was not noted on regular examination. Pap smear was normal. Her personal, family and gynaecological history with two normal vaginal deliveries was unremarkable. Menstrual cycle was regular, 28/4 days. She is office worker and was not exposed to any hazards. Transvaginal ultrasonography did not show any abnormality. CT imaging or magnetic resonance imaging (MRI) was not administrated. The decision for local excision with clear margins was made and surgery was performed under general anaesthesia. The procedure was simple and post-operative recovery unremarkable. She was discharged home second postoperative day. Pathological examination showed that the excised mass was tumor. The excised tumor was rounded, well circumscribed and measured approximately $5 \mathrm{~cm}$ in diameter. Material was brownish, in the cross section whitish and medium firm. Histologically, the tumor was composed of round to spindle cells, eosinophilic cytoplasm with interspersed visible myxomatosus areas, multinucleated cells and mast cells (Figure 1). Protruded blood vessels surrounded by previously described cells were seen. Immunohistochemistry tumor cells were desmin + (Figure 2), vimentin + and most of them were mildly positive for CD 34 (Figure 3), while SMA and Caldesmon negative. Onetwo mitosis was found in 50 fields under high magnification (40X). Diagnosis of angiomyofibroblastoma was established.

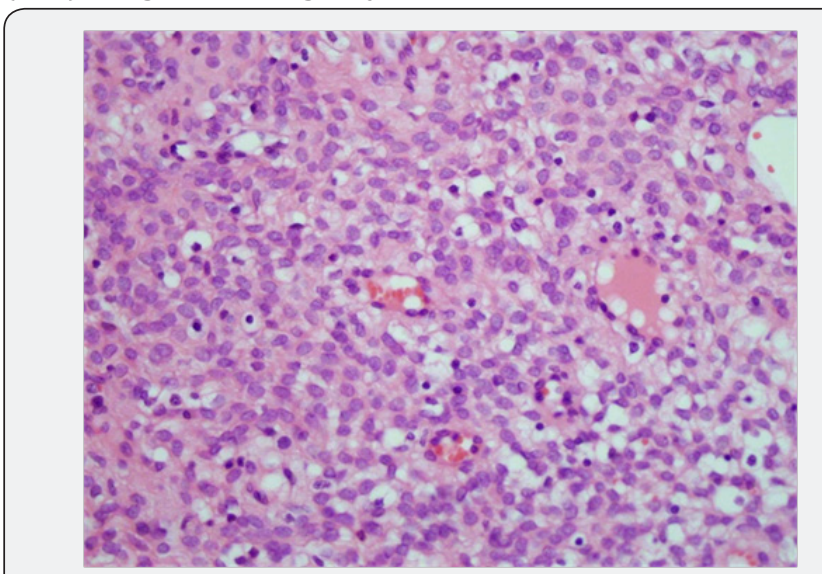

Figure 1: Tumor was composed of bland-looking cells ranging from ovoid- to spindle shaped cells. (HE, x40). 

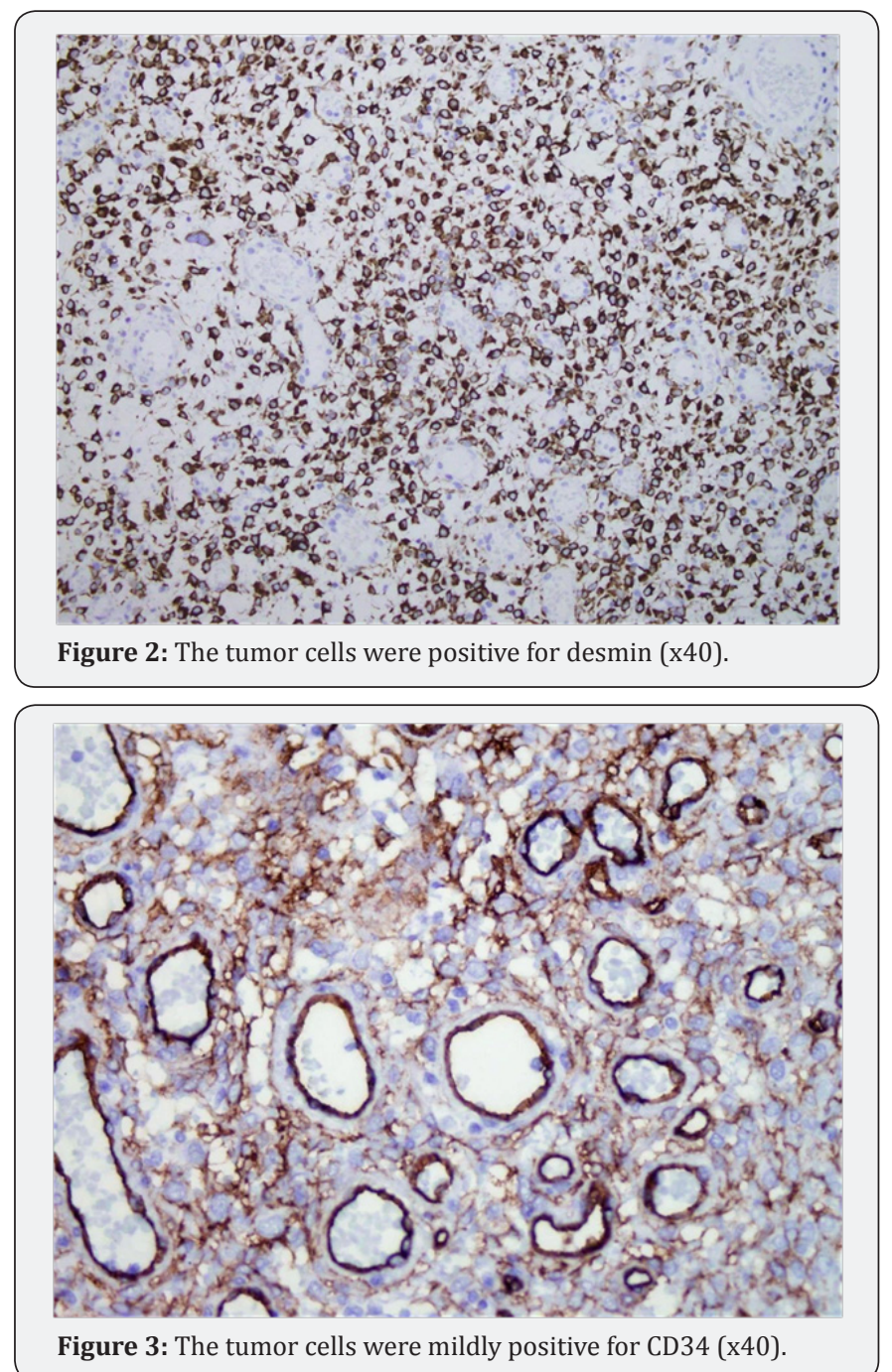

Discussion

Angiomyofibroblastoma (AMF) is a rare, benign, softtissue tumor that belongs to the group of genital mesenchymal tumors, which was first described in 1992 [1]. Macroscopic appearance describes rounded solid mass while microscopy shows myofibroblastic differentiation and represents neoplastic proliferation of stromal cells. Incidence of AMF is unknown; no statistical data are available, due to its rareness. Cause and pathogenesis are also unknown. Reviewing the literature, AMF is the most frequently described in women between 20 and 50 years of age, in vulvovaginal region [1,2] with only few reports describing AMF in male patients in inguinoscrotal region $[5,10]$.

$\mathrm{AMF}$ is mostly benign. In literature only one case with sarcomatous transformation has been reported [11]. A rare lipomatous variant of AMF with local invasion has also been reported [12,13]. Most of the reported cases of AMF present it as a painless neoplasm located in the lower female genital tract, such as the vulva and vagina [4]. Those AMF tumors that arise in the cervix, uteri or urethral region sometimes are present with obstructive symptoms [14]. Giant tumors in dimension ranged from 3.8 to $25 \mathrm{~cm}$ are extremely rare, difficult to diagnose and usually grow insidiously in the pelvis, iliac fossa or peritoneal cavity [15-18].

All described cases of the pelvic AMFs were well demarcated [6,7,15-18]. Different imaging methods help in diagnostics of AMF (perineal ultrasonography, CT imaging, MR imaging). On perineal ultrasonography, AMFs appear as a soft-tissue mass with inhomogeneous mixed echogenicity. That echogenicity corresponds to the cellular inhomogenecity found on histopathological examination. The ultrasonographic characteristics of AMFs may help to differentiate AMF from other mesenchymal neoplasms [19]. On CT imaging, AMFs show moderate- to-strong enhancement, which may reflect the prominent vascularity of these tumors [19].

The AMF and cellular angiofibroma, both have a characteristic vascular network and spindle cell component, so the difference between them is relatively subtle. Cellular angiofibroma is characterized by the presence of spindle cell lipomas and thickwalled vessels, meanwhile cellular angiofibromas lack estrogen receptors and progesteron receptors [12].

Aggressive angiomyxoma (AAM) is easily confused with AMF because it shares many characteristics with AMF (age at presentation, location, clinical manifestations and pathological entities). The AAM is presented as a malignant, locally infiltrative, non-metastasizing stromal neoplasm and have strong tendency to recur. Although on microscopic examination, AMFs generally show much higher cellularity, more numerous blood vessels and more frequent plump or short spindle-shaped cells, AAM cells are sparsely and diffusely distributed, without the characteristics of alternating density and aggregation around small blood vessels. AAMs show more distinctive myxoid degeneration than AMFs $[1,20]$. AMFs main characterics are expression ofvimentin, desmin and CD34. This characteristic of suggest that AMF is an undifferentiated mesenchymal tumor with preferential myofibroblastic differentiation. Most AAMs are positive for estrogen and progesterone receptors, and some show positive expresion of desmin. The recent clinical studies show that after surgical treatment, 30\% of AAMs have been found to relapse within 2 years [1]. The immunohistochemical (IHC) markers of AMF have similiar morphology to those of AAM, so the differentiation between these two tumors is mostly based on the appearance of the tumor margins (AMFs are well demarcated).

As AMF is a commom perineum-site-specific stromal tumour, a benign neoplasm of dendritic fibromyxolipoma (DFML) in rare sites such as perineum regions should be differentiated from AMF. Zhang XJ et al. [21] recently reported the case of a woman with a slow-growing, painless, subcutaneous tumor in the right inguinal and perineal regions which was diagnosed as DFML lately. DFML is histologically mainly composed of a proliferation of small spindle or stellate cells that are variably admixed with mature adipose tissue embedded within an abundant myxoid and collagenized stroma. The spindle and stellate cells are 
immunohistochemically strongly positive for vimentin, CD34, and bcl-2 antibodies but not for smooth muscle actin and desmin. The histopathological and immunohistochemical findings mentioned above can easily distinguish AMF from DFML.

Inflammatory myofibroblastic tumor (IMT) has myofibroblastic differentiation and these rare benign [22] lesions are predominately located in the lung. Microscopic examination reveals a proliferation of regular myofibroblastic spindle cells arranged within a fibrous, myxoid or calcified stroma, associated with an inflammatory component of lymphocytes and plasmacytes but without blood vessels [22]. Immunohistochemical studies show reactivity for vimentin, smooth muscle actin and ALK.

AMF should be differentiated from other tumor-like lesions of the vulvovaginal region such as Bartholin cysts, benign lipoma, fibroepithelial stromal polyps, cellular angiofibromas, carcinoma of cervics, condyloma accuminata, endocervical polyp and pedunculated submucous myoma $[1-4,12,13]$.

AMF shows no affinity for infiltrative growth and treatment is total local excision with clear margins. There are no data of incidences of recurrences or metastasis after complete excision [23].

\section{Conclusion}

AMF is extremely rare, benign non-recurring tumor of vulvovaginal and ingvinoscrotal region. Preoperative diagnosis from other soft-tissue tumors is very difficult. The combination of imaging data, histological and IHC findings can confirm the diagnosis. The total local excision with clear margins is choice of treatment.

\section{References}

1. Fletcher CD, Tsang WY, Fisher C, Lee KC, Chan JK (1992) Angiomyofibroblastoma of the vulva. A benign neoplasm distinct from aggressive angiomyxoma. Am J Surg Pathol 16(4): 373-382.

2. Babala P,Biro C, Klacko M, Miklos P, Ondrus D (2011) Angiomyofibroblastoma of the cervix uteri: a case report. Klin Onkol 24(2): 133-136.

3. Mortele KJ, Lauwers GJ, Mergo PJ, Ros PR (1999) Perineal angiomyofibroblastoma: CT and MR findings with pathologic correlation. J Comput Assist Tomogr 23(5): 687-689.

4. Naheed S, Upadhyay K, Pradeep K (2011) Angiomyofibroblastoma of the vulva. J Obstet Gynaecol 31: 554-555.

5. Lee SH,YangJW, Do JM,Seo DH, JungJH, etal. (2010) Angiomyofibroblastomalike tumor of the scrotum. Korean J Urol 51(5): 365-367.
6. Lim KJ, Moon JH, Yoon DY, Cha JH, Lee IJ, etal. (2008) Angiomyofibroblastoma arising from the posterior perivesical space: a case report with MR findings. Korean J Radiol 9(4): 382-385.

7. Qiu P, Wang Z, Li Y, Cui G (2014) Giant pelvic angiomyofibroblastoma: case report and literature review. Diagn Pathol 3(9):106.

8. Nasu K, Fujisawa K, Takai N, Miyakawa I (2002) Angiomyofibroblastoma of the vulva. Int J Gynecol Cancer 12(2): 228-231.

9. Saleh MM, Yassin AH, Zaklama M (2007) Recurrent angiomyofibroblastoma of the vagina: a case report. Eur J Gynaecol Oncol 28(4): 324.

10. Ding G, Yu Y, Jin M, Xu J, Zhang Z (2014) Angiomyofibroblastoma-like tumor of the scrotum: a case report and literature review. Oncol Lett 7(2): 435-438.

11. Nielsen GP, Young RH, Dickersin GR, Rosenberg AE (1997) Angiomyofibroblastoma of the vulva with sarcomatous transformation ("angiomyofibrosarcoma"). Am J Surg Pathol 21(9): 1104-1108.

12. Laskin WB, Fetsch JF, Tavassoli FA (1997) Angiomyofibroblastoma of the female genital tract: analysis of 17 cases including a lipomatous variant. Hum Pathol 28(9): 1046-1055.

13. VoraS, GabaND,StamatakosMD(2011) Lipomatousangiomyofibroblastoma: a case report of a unique vulvar mass. J Reprod Med 56: 347-350.

14. Kitamura H, Miyao N, Sato Y, Matsukawa M, Tsukamoto T, et al. (1999) Angiomyofibroblastoma of the female urethra. Int J Urol 6(5): 268-270.

15. QuinteroC,Sasken H,HouckKL,HernandezE (2007) Angiomyofibroblastoma of the retroperitoneum: a case report. J Reprod Med 52: 741-744.

16. Menendez SP, Villarejo CP, Padilla VD, Munoz AV, Gonzalez LL. et al. (2010) Angiomyofibroblastoma of the right ischiorectal fosa. Cir Cir 78: 448-450.

17. Lim KJ, Moon JH, Yoon DY, Cha JH, Lee IJ, etal. (2008) Angiomyofibroblastoma arising from the posterior perivesical space: a case report with MR findings. Korean J Radiol 9(4): 382-385.

18. Kobayashi T, Suzuki K, Arai T, Sugimura H (1999) Angiomyofibroblastoma arising from the fallopian tube. Obstet Gynecol 94(5): 833-834.

19. Kim SW, Lee JH, Han JK, Jeon S (2009) Angiomyofibroblastoma of the vulva: sonographic and computed tomographic findings with pathologic correlation. J Ultrasound Med 28: 14170-1420.

20. Steeper TA, Rosai J (1983) Aggressive angiomyxoma of the female pelvis and perineum. Am J Surg Pathol 7(5): 463-475.

21. Report of nine cases of a distinctive type of gynecologic soft-tissue neoplasm. Am J Surg Pathol 7: 463-475.

22. Zhang XJ, Zhou S, Nie K, Chen DF, Kui GJ, et al. (2013) Dendritic fibromyxolipoma in the right inguinal and perineum regions: a case report and review of the literature. Diagn Pathol 8: 157-163.

23. Nawal H, Laila C, Mohammed R, Meryem B, Sara B, et al. (2012) A rare tumor of the lung: inflammatory myofibroblastic tumor. Diagn Pathol 7: 83-86.

24. Stewart C (2009) Angiomyofibroblastoma of the vagina. Pathology 41(2): 199-200. 